options. The cost-effectiveness of each chosen option will then be assessed.

Conclusions We have developed a provisional framework for developing policy options, initially for the prevention of CVD and diabetes. This is based on local epidemiological data, an assessment of the socio-political-cultural context and cost effectiveness. Policy makers are involved throughout, and will be presented with costed policy options along with their potential consequences. Implemented options will then be evaluated. The framework represents a "policy effectiveness-feasibility loop", analogous to Tugwell's clinical effectiveness loop. The impact of this approach, and its potential generalisability, will be rigorously evaluated.

\section{SYSTEMATIC REVIEW: THE USE OF RESEARCH EVIDENCE BY PUBLIC HEALTH POLICY-MAKERS}

doi:10.1136/jech.2010.120956.52

L Orton, F Lloyd-Williams, D Taylor-Robinson, M O'Flaherty, S Capewell. Division of Public Health, School of Population Community and Behavioural Sciences, University of Liverpool, Liverpool, UK

Objective To review: the process of public health policy-making; variations in the extent of research evidence used; other influencing factors; and barriers to and facilitators of the use of research evidence.

Design Systematic review of empirical studies reporting data on policy-making in public health.

Data Sources Databases searched: MEDLINE, SCOPUS, PsychInfo, CINAHL, The Social Science Citation Index, The Science Citation Index, The Arts and Humanities Citation Index, Applied Social Sciences Index and Abstracts, Database of Reviews of Effects, Cochrane Database of Systematic Reviews, DoPHER, the Campbell Library, and the Cochrane Register of Controlled trials. Other sources: screening of organisational websites, contacting key informants and scrutinising the bibliographies of included studies.

Review methods Two reviewers independently assessed studies for inclusion; extracted data and assessed methodological quality using predesigned forms. Disagreements were resolved by consensus or by recourse to a third reviewer. Data were synthesised as a narrative review.

Results 1216 articles were retrieved. Following screening 18 studies were included: 13 qualitative studies, four surveys and one literature review. Participants included 1200 policy-makers, 72 researchers, and 174 people involved in both activities. Studies were set in a range of country and policy-making settings. Methodological quality was mixed. The process of policy-making varies widely between settings, and is viewed differently by key players. An extensive range of types of research evidence are used in policymaking. However, it has only an indirect impact and competes with many other influences. Barriers to the use of research evidence are well-described and include: policy-makers' perceptions of research evidence; the gulf between researchers and policy-makers; the culture in which policy-makers work; competing influences on policy-making; and practical constraints. Ways of overcoming these barriers are less well known, and include: research targeted at the needs of policy-makers; research clearly highlighting key messages; and capacity building. There is almost no evidence on the role of research evidence in addressing health inequalities, a key aim of public health policy.

Conclusions Action is required by both policy-makers and researchers to address the barriers identified in this systematic review. There is an urgent need for evidence on the best approaches to incorporating research evidence in public health policy, particularly that considering the complex effects on health inequalities.
053 PROTECTION AND PUBLIC HEALTH: POPULATION EVIDENCE FROM THE EU, 1980-2003

doi:10.1136/jech.2010.120956.53

${ }^{1,2} \mathrm{D}$ Stuckler, ${ }^{3} \mathrm{~S}$ Basu, ${ }^{2} \mathrm{M}$ McKee. ${ }^{1}$ Department of Sociology, Oxford University, Oxford, UK; ${ }^{2}$ London School of Hygiene and Tropical Medicine, Department of Public Health and Policy, London, UK; ${ }^{3}$ Department of Internal Medicine, University of California at San Francisco, San Francisco, California, USA

Objectives To assess the effect of social spending on population health.

Design Multivariate regression analysis was performed to investigate the relationship between age-standardised cause-specific mortality rates and social spending. Mortality data were collected from the European Health for All Database 2009 edition. Social spending per capita in purchasing-power-parity were taken from the OECD Social Expenditure Database (including family support, oldage pensions, healthcare, unemployment benefits, active labour market programmes, and support for people with disabilities). These relationships were compared with gross domestic product per capita (GDP), general government spending per capita (including prisons, education and defence), and healthcare spending per capita. Models also included controls for country- and period-fixed effects and expenditure was adjusted for inflation and purchasing power parity. Setting 13 EU countries, 1980-2003: Belgium (only to 1998), Denmark, Finland, France, Germany, Greece, Ireland, Italy, Netherlands, Spain, Sweden and the UK.

Participants $\mathrm{n} / \mathrm{a}$.

Main outcome measure Age-standardised all-cause and cause-specific mortality rates.

Results Each additional US\$100 increase in social spending per capita is significantly associated with a $0.99 \%$ reduction in all-cause, age-standardised mortality rates $(p<0.001)$. Healthcare spending per capita had no effect on all-cause mortality rates. Consistent with previous findings, we observed each US\$100 increase in GDP was associated with a $0.28 \%$ fall in all-cause mortality rates $(p<0.001)$, about one-fourth of the magnitude of the association of social welfare with mortality. After adjusting for social welfare spending, the association of GDP with all-cause mortality was reduced by over half ( $\beta=-0.11 \%, p=0.004)$. Healthcare spending was not associated with mortality. However, higher social welfare spending was significantly associated with reductions in alcohol-related deaths, cardiovascular disease and tuberculosis; findings which are biologically plausible.

Conclusion Reducing social spending could increase mortality rates. The majority of the benefits of wealth to health appears to be determined by the extent to which resources are invested in social welfare and healthcare systems. Investments in social protection seem to have greater protective effects on health than investments in health care. Stronger links between health and labour ministries offer an opportunity to take advantage of such positive synergies, especially important to protecting health during times of austerity.

\section{Ethnicity}

\section{ANOTHER HISPANIC PARADOX? THE HEALTH BENEFITS OF HISPANIC COMMUNITIES FOR NON-HISPANIC MOTHERS AND INFANT}

doi:10.1136/jech.2010.120956.54

R J Shaw, K E Pickett. Department of Health Sciences, University of York, York, UK

Background and Objectives In the US, Hispanic mothers have rates of infant mortality and low birthweight that are comparable to nonHispanic White mothers despite being more likely to live in socioeconomically deprived areas. This well known phenomenon is 
termed the Hispanic paradox. Recent research suggests that this phenomenon may be partly explained by the areas in which Hispanic people live. Hispanic mothers living in counties with a high proportion of Hispanic people (Hispanic density) have lower rates of infant mortality and smoking during pregnancy. In this paper, we investigate whether or not Hispanic density is associated with better birth outcomes for mothers of other ethnicities.

Design Multilevel analysis of the US Linked Birth and Infant Death Dataset 2000 and US census data at county level.

Setting USA.

Participants 2274247 White and 581151 Black non-Hispanic mothers of singleton births.

Main outcome measures Infant mortality, low birthweight, preterm delivery and maternal smoking during pregnancy.

Results Living in counties with a higher percentage of Hispanic residents was associated with reduced risk of all outcomes for nonHispanic White and Black mothers and infants in analyses adjusting for individual and area level socio-demographic characteristics. The reduction in odds was greatest for maternal smoking during pregnancy. White mothers living in counties where more than half the residents were Hispanic had their odds of smoking during pregnancy reduced by approximately $80 \%$ (OR $0.1995 \%$ CI 0.11 to 0.33 ), relative to comparable mothers living at Hispanic densities of 0 to $1 \%$. Similar reductions in risk of maternal smoking during pregnancy were found for Black mothers (OR 0.14 $95 \%$ CI 0.14 to 0.51 ). Infant mortality was reduced by approximately a third for both Black and White mothers living in counties with a high proportion of Hispanic residents. In addition, higher Hispanic density was associated with modest but significant reductions in the risks of preterm delivery and low birthweight.

Conclusions Living in Hispanic communities appears to have health benefits for those of non-Hispanic origin.

\section{COMPARISON OF DISTRIBUTIONS OF COMMON INDICATORS OF SOCIO-ECONOMIC POSITION BY ETHNICITY AND MIGRATION: PRELIMINARY FINDINGS FROM THE BORN IN BRADFORD BIRTH COHORT STUDY}

doi:10.1136/jech.2010.120956.55

${ }^{1} \mathrm{~L}$ Fairley, ${ }^{2} \mathrm{~N}$ Small, ${ }^{3} \mathrm{D}$ A Lawlor, ${ }^{1} \mathrm{~J}$ Wright. ${ }^{1}$ Bradford Institute for Health Research, Bradford Royal Infirmary, Bradford, UK; ${ }^{2}$ School of Health Studies, University of Bradford, Bradford, UK; ${ }^{3}$ MRC Centre for Causal Analyses in Translational Epidemiology, University of Bristol, Bristol, UK

Objective To describe and compare the distributions, including extent of missing data, in measures of socio-economic position (SEP) between women of white British and Pakistani ethnicity. We also compare distributions of measures of SEP between Pakistani women born in UK and those born in Pakistan and also compare distributions by the woman's age at migration to the UK.

Design The Born in Bradford birth cohort study recruits pregnant women at 26-28 weeks gestation when they complete a baseline questionnaire; approximately half these women are of Pakistani origin.

Setting Bradford, UK.

Participants Data are currently available for 2005 White British and 2444 Pakistani women.

Main outcome measure Indicators of SEP included in these analyses are: the woman's education and employment, her partner's education and employment and household income.

Results $57 \%$ of the Pakistani ethnicity women were born in Pakistan and there was a bimodal distribution of age at migration to the UK with peaks at ages 1 and 18 years. 92\% of the White British women were, or had been, in paid employment compared to $51 \%$ of the
Pakistani women. This figure was $82 \%$ for UK-born Pakistani women, $73 \%$ for those born in Pakistan who moved aged 5 or under and $22 \%$ for those who moved after the age of 5 . Overall $23 \%$ of women reported that they didn't know their family income; this varied by ethnicity and country of birth (8\% for White British women, 21\% for UK-born Pakistani women and 49\% for those born in Pakistan). The percentage of women reporting an income of less than $£ 20000$ was highest in Pakistani women, while the percentage of women reporting an income of $£ 20000$ and over was highest for the White British women. 11\% of White British women had no educational qualifications compared to $22 \%$ of Pakistani women; this figure was lowest for UK-born Pakistani women. The percentage of women with degree level education was higher in Pakistani than White British women (26\% and 19\%, respectively) and was similar for all Pakistani women irrespective of migration history. The percentage of women's partners with no qualifications was similar between ethnic groups, however the percentage of partners with degree level education was higher for the Pakistani women.

Conclusion These differences in the distributions of SEP measures by ethnicity and migration are important to understand health inequalities and for ensuring appropriate adjustment of SEP confounding.

\section{SOCIO-DEMOGRAPHIC, HEALTH STATUS, PSYCHO-SOCIAL AND LIFESTYLE PREDICTORS OF SELF-RATED HEALTH IN THE ALL IRELAND TRAVELLER HEALTH STUDY}

doi:10.1136/jech.2010.120956.56

${ }^{1} \mathrm{~J}$ Whelan, ${ }^{1} \mathrm{P}$ Fitzpatrick, ${ }^{1} \mathrm{~S}$ Abdalla, ${ }^{1} \mathrm{~F}$ Cronin, ${ }^{1} \mathrm{~A}$ Drummond, ${ }^{1} \mathrm{~L}$ Daly, ${ }^{1} \mathrm{~K}$ Frazier, ${ }^{1} \mathrm{~N}$ A Hamid, ${ }^{1} \mathrm{C}$ Kelly, ${ }^{1} \mathrm{C}$ McGorrian, ${ }^{1} \mathrm{R}$ G Moore, ${ }^{1} \mathrm{R}$ NicCharthaigh, ${ }^{1} \mathrm{~B}$ O'Shea, ${ }^{1} \mathrm{D}$ O'Mahony, ${ }^{1} \mathrm{~B}$ Quirke, ${ }^{2} \mathrm{~A}$ Staines, ${ }^{1} \mathrm{D}$ Staines, ${ }^{2} \mathrm{M} \mathrm{R}$ Sweeney, ${ }^{1} \mathrm{~J}$ Turner, ${ }^{1} \mathrm{C} C$ Kelleher. ${ }^{1}$ UCD School of Public Health, Physiotherapy and Population Science, University College Dublin, Belfield, Dublin, Republic of Ireland; ${ }^{2}$ School of Nursing, Dublin City University, Dublin, Republic of Ireland

Background Irish Travellers have a lower life expectancy than the general Population. Objective health-status indices are consistently poorer in Traveller and Gypsy populations than in other comparably deprived social groups. Self-rated Health (SRH) is established as a valid indicator of objective health status.

Methods As part of an all-Ireland census of 10618 Traveller families both North and South (response rate $80 \%$ overall) employing a novel audio-visual computer interview methodology with peer researchers to overcome literacy barriers, a personal, structured interview was conducted with a random $20 \%$ sub-sample of adults $(n=2065,43.5 \%$ male). This analysis describes predictors of SRH in those adults, within health domains such as socio-demographic, environmental, lifestyle and psychosocial factors (including those related to culture/ identity, and to social capital such as experiences of discrimination). Six models were constructed initially for each domain separately and then a final backwards stepwise logistic regression model was chosen that included 36 potential predictors from all domains of health, with self rated health as a outcome variable dichotomised into categories good (excellent, very good or good) vs fair or poor.

Results SRH was rated as excellent, good or very good by $82.6 \%$ of respondents and was age-related. Those who reported limiting disability/long-term illness were excluded. Each separate domain model showed variables predictive of SRH. On adjustment for agegroup and sex in the social capital model for instance, experience of discrimination (OR 0.5, $\mathrm{p}=0.02$ ), low levels of trust (OR 0.6, $p=0.04$ ) and worry about getting fair treatment (OR 0.6, $p=0.007$ ) were independently inversely associated with good SRH. In the final model, independent positive predictors of good SRH were having a flush toilet ( $\mathrm{OR} 2.2, \mathrm{p}=0.02$ ), considering where you live to be 